\title{
Short note on comparing stacking modelling versus Cannistraci- Hebb adaptive network automata for link prediction in complex networks
}

\author{
Alessandro Muscoloni ${ }^{1}$ and Carlo Vittorio Cannistraci ${ }^{1,2, *}$ \\ ${ }^{1}$ Center for Complex Network Intelligence $(\mathrm{CCNI})$ at Tsinghua Laboratory of Brain and Intelligence \\ (THBI), Department of Bioengineering, Tsinghua University, 160 Chengfu Rd., SanCaiTang Building, \\ Haidian District, 100084, Beijing, China. \\ ${ }^{2}$ Center for Systems Biology Dresden (CSBD), Pfotenhauerstr. 108, 01307, Dresden, Germany \\ *Corresponding author: Carlo Vittorio Cannistraci (kalokagathos.agon@gmail.com)
}

Keywords: complex networks, network models, link prediction, automata theory, network automata, Cannistraci-Hebb, stacking modelling

\begin{abstract}
Link prediction is an iconic problem in complex networks because deals with the ability to predict nonobserved existing or future parts of the network structure. The impact of this prediction on real applications can be disruptive: from prediction of covert links between terrorists in their social networks to repositioning of drugs in molecular diseasome networks.

Here we compare: (1) an ensemble meta-learning method (Ghasemian et al.), which uses an artificial intelligence (AI) stacking strategy to create a single meta-model from hundreds of other models; (2) a structural predictability method (SPM, Lü et al.), which relies on a theory derived from quantum mechanics and does not assume any model; (3) a modelling rule named Cannistraci-Hebb $(\mathrm{CH}$, Muscoloni et al.), which relies on one brain-bioinspired model adapting to the intrinsic network structure.

We conclude that brute-force stacking of algorithms by Al does not perform better than (and is often significantly outperformed by) SPM and one simple brainbioinspired rule such as $\mathrm{CH}$. This agrees with the Gödel incompleteness: stacking is optimal but incomplete, you cannot squeeze out more than what is already in your features. Hence, we should also pursue Al that resembles human-like physical 'understanding' of simple generalized rules associated to complexity. The future might be populated by Al that 'steals for us the fire from Gods', towards machine intelligence that creates new rules rather than stacking the ones already known.
\end{abstract}

\section{Short Note}

From nests to nets intricate wiring diagrams surround the birth and death of life and we, as humans, wonder what the secret rules behind such elegant network architectures are. If nature adopts connectivity to shape complexity, humans might adopt their intelligence, or an artificial one, to make sense of it. To predict structural complexity in a network: should we act as small Prometheans, exploiting creativity to 'steal the fire from Gods' and search, as physicists aim, for that accurate but simple, 
elegant unique rule that makes sense of 'everything'? Or should we ask an artificial intelligence (Al) to stack for us hundreds of inaccurate human-made rules to make a new one that optimally summarizes them together? Perhaps, none of these two solutions is what the future holds for us. Nevertheless, Ghasemian et al. ${ }^{1}$ embrace the second option and propose a stacking model: a machine learning method for link prediction based on ensemble meta-learning, using artificial intelligence to create a single meta-model from hundreds of other models. Analysing the results on 550 realworld networks, Ghasemian et al. conclude that they obtain a state-of-the-art algorithm achieving nearly optimal prediction ${ }^{1}$.

Here, we suggest that the scientific community might benefit to interpret the results of Ghasemian et al. when their algorithm is compared with other state-of-the-art algorithms which largely differ from ensemble meta-learning. One is structural predictability method $(\mathrm{SPM})^{2}$, which relies on a theory derived from quantum mechanics that exploits the first-order perturbation of the graph adjacency matrix. SPM takes a way opposite to that of stacking: it simply does not assume any model.

Interestingly, also brain and life science theories contribute to inspire revolutionary algorithms for link prediction ${ }^{3}$. After Daminelli et al. ${ }^{4}$ and Kovács et al. ${ }^{5}$ elucidated the importance to build models that rely on paths of length 3 inducing quadrangular closure ${ }^{4}$, Muscoloni et al. ${ }^{6}$ proposed a general theory of adaptive network automata that includes a simple modelling rule named Cannistraci-Hebb $(\mathrm{CH})$. $\mathrm{CH}$ is inspired by neuro-plasticity concepts and scales adaptively on paths of different length, in relation with the intrinsic network structure ${ }^{6}$.

It might be enlightening to compare a method that stacks multiple models versus SPM, which is model-free, and $\mathrm{CH}$-adaptive ( $\mathrm{CHA})$, which relies on one model adapting to the intrinsic network structure. Using the 550 networks of Ghasemian et al. ${ }^{1}$, we report the results according to mean performance (Figure 1) and win rate (Figure 2) for Precision, AUC-PR and AUC-ROC, following evaluation strategies previously employed ${ }^{2,7}$.

Brute-force stacking of algorithms by Al does not perform better than (and is often significantly outperformed by) SPM and one simple brain-bioinspired rule such as $\mathrm{CH}$. This agrees with the Gödel incompleteness: stacking is optimal but incomplete, you cannot squeeze out more than what is already in your features. Hence, we should also pursue Al that resembles human-like physical 'understanding' of simple generalized rules associated to complexity ${ }^{8}$. The future might be populated by Al that 'steals for us the fire from Gods', towards machine intelligence that creates new rules rather than stacking the ones already known.

\section{Acknowledgments}

We thank Umberto Michieli and Stephan Wolfgang Grill for proofreading the article and for offering suggestions.

\section{References}

1. Ghasemian, A., Hosseinmardi, H., Galstyan, A., Airoldi, E. M. \& Clauset, A. Stacking models for nearly optimal link prediction in complex networks. Proc. Natl. Acad. Sci. U. 
S. A. (2020) doi:10.1073/pnas.1914950117.

2. Lü, L., Pan, L., Zhou, T., Zhang, Y.-C. \& Stanley, H. E. Toward link predictability of complex networks. Proc. Natl. Acad. Sci. 112, 2325-2330 (2015).

3. Cannistraci, C. V. Modelling Self-Organization in Complex Networks Via a BrainInspired Network Automata Theory Improves Link Reliability in Protein Interactomes. Sci. Rep. 8, 15760 (2018).

4. Daminelli, S., Thomas, J. M., Durán, C. \& Cannistraci, C. V. Common neighbours and the local-community-paradigm for topological link prediction in bipartite networks. New J. Phys. 17, 113037 (2015).

5. Kovács, I. A. et al. Network-based prediction of protein interactions. Nat. Commun. (2019) doi:10.1038/s41467-019-09177-y.

6. Muscoloni, A., Michieli, U. \& Cannistraci, C. V. Adaptive Network Automata Modelling of Complex Networks. Preprints (2020) doi:10.20944/preprints202012.0808.v2.

7. Zhou, T., Lee, Y.-L. \& Wang, G. Experimental analyses on 2-hop-based and 3-hopbased link prediction algorithms. Phys. A Stat. Mech. its Appl. 564, 125532 (2021).

8. Seif, A., Hafezi, M. \& Jarzynski, C. Machine learning the thermodynamic arrow of time. Nat. Phys. (2021) doi:10.1038/s41567-020-1018-2.

9. Saito, T. \& Rehmsmeier, M. The precision-recall plot is more informative than the ROC plot when evaluating binary classifiers on imbalanced datasets. PLoS One (2015) doi:10.1371/journal.pone.0118432.

10. Yang, Y. et al. Evaluating link prediction methods. Knowl. Inf. Syst. 45, 751-782 (2015). 


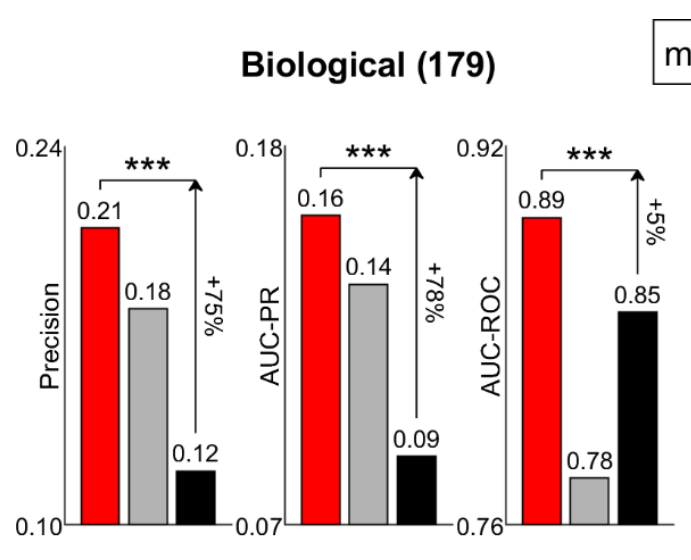

Informational (18)

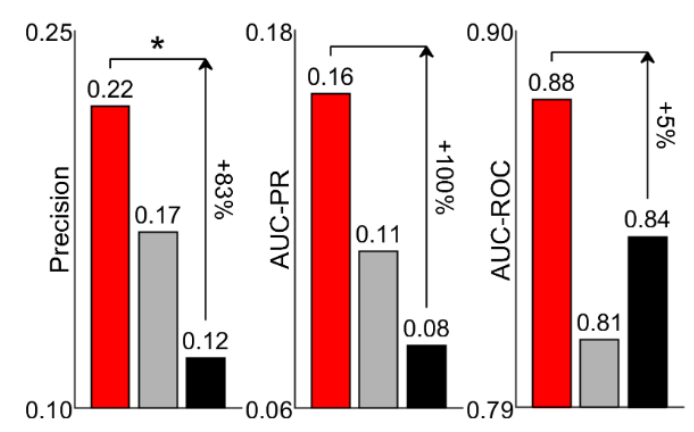

Technological (70)

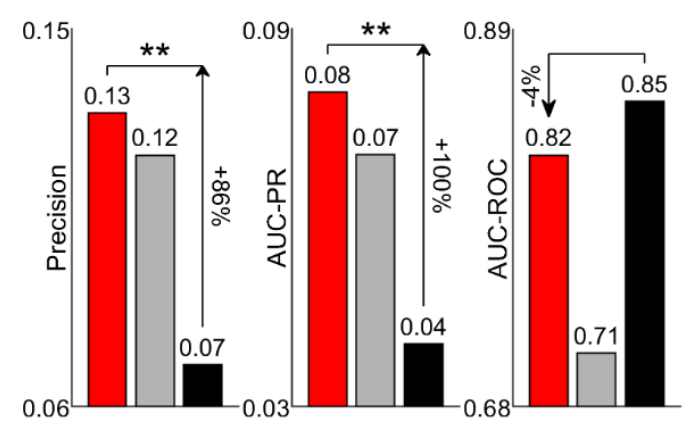

Economic (124)

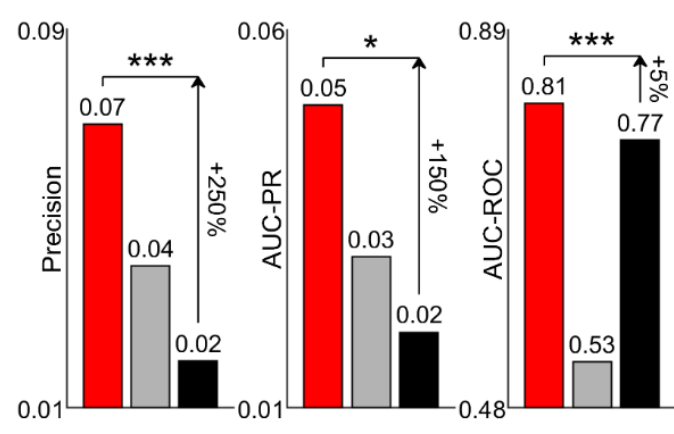

Social (124)

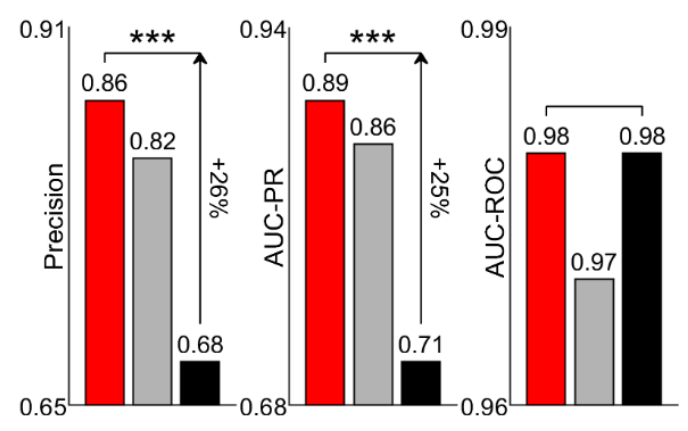

Transportation (35)

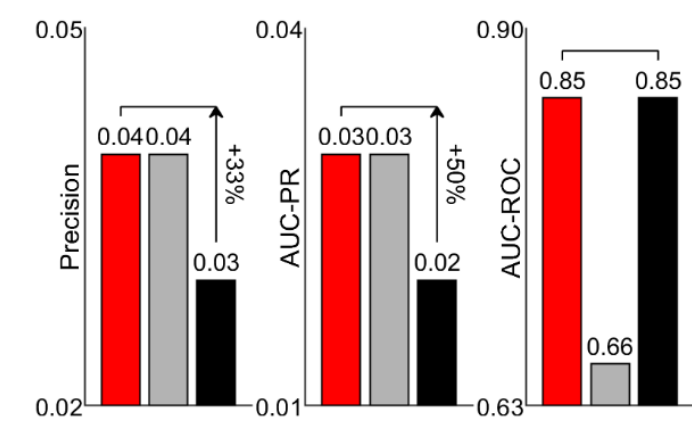

$\mathrm{CHA}$

SPM

stacking

Figure 1. 550 real-world networks of Ghasemian et al. ${ }^{1}$ are considered. Given a network X, $10 \%$ of links are removed uniformly at random (keeping the network as one connected component), obtaining a reduced network $X^{\prime}=X-R$, where $R$ is the set of removed links. For evaluating each algorithm, the reduced network $X^{\prime}$ is given in input, obtaining in output a ranking of the nonobserved links in $X^{\prime}$ by decreasing likelihood scores. The prediction performance is evaluated using Precision, AUC-PR and AUC-ROC, considering as positive samples the set $R$ of links previously removed. This procedure is repeated 10 times and the mean value indicates the performance of the algorithm on the network X. The barplots report, for each network domain (biological, economic, informational, social, technological, transportation) and for each evaluation measure, the mean performance over the networks (whose number is indicated in brackets) of that domain for CHA, SPM and stacking. The arrows highlight the percentage of improvement of CHA with respect to stacking as $\frac{\text { CHA-stacking }}{\text { stacking }} \cdot 100$. The asterisks represent the $\mathrm{p}$-value significance of the permutation test for the mean, comparing the performances of $\mathrm{CHA}$ and stacking on the networks of that domain. AUC-ROC is included for completeness, although studies showed that this measure is misleading for unbalanced prediction tasks ${ }^{9}$ such as link prediction and that in its lieu AUC-PR should be preferred ${ }^{10}$. 

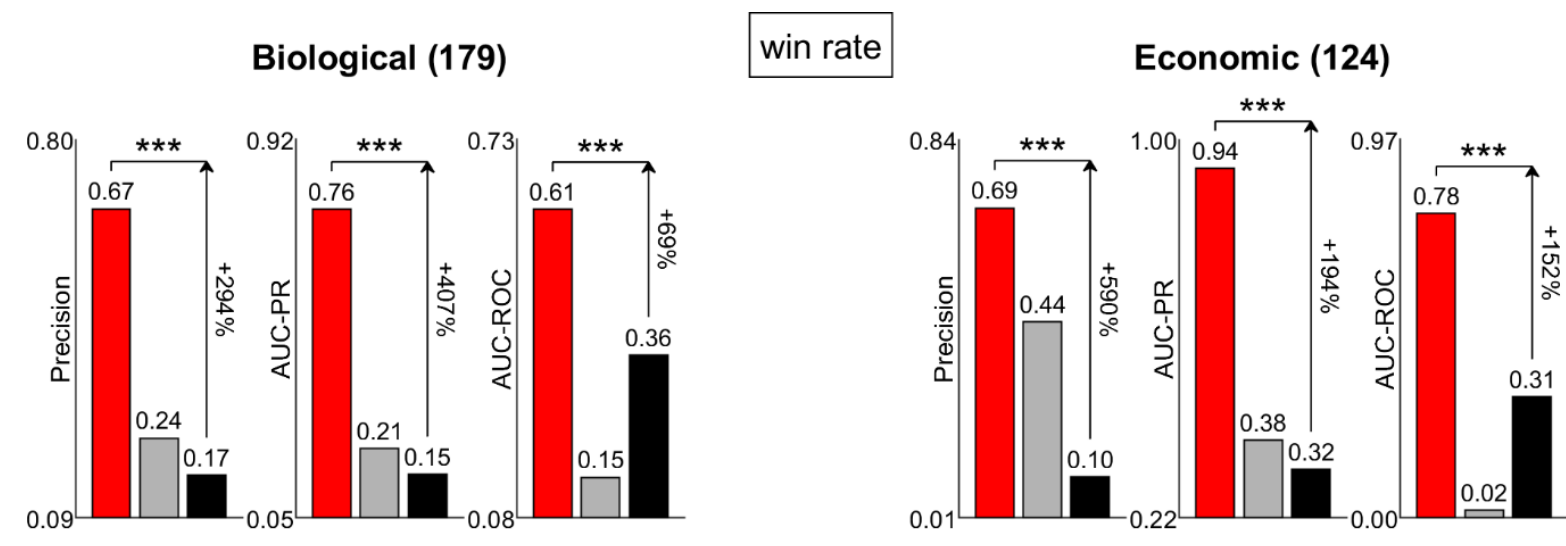

Informational (18)
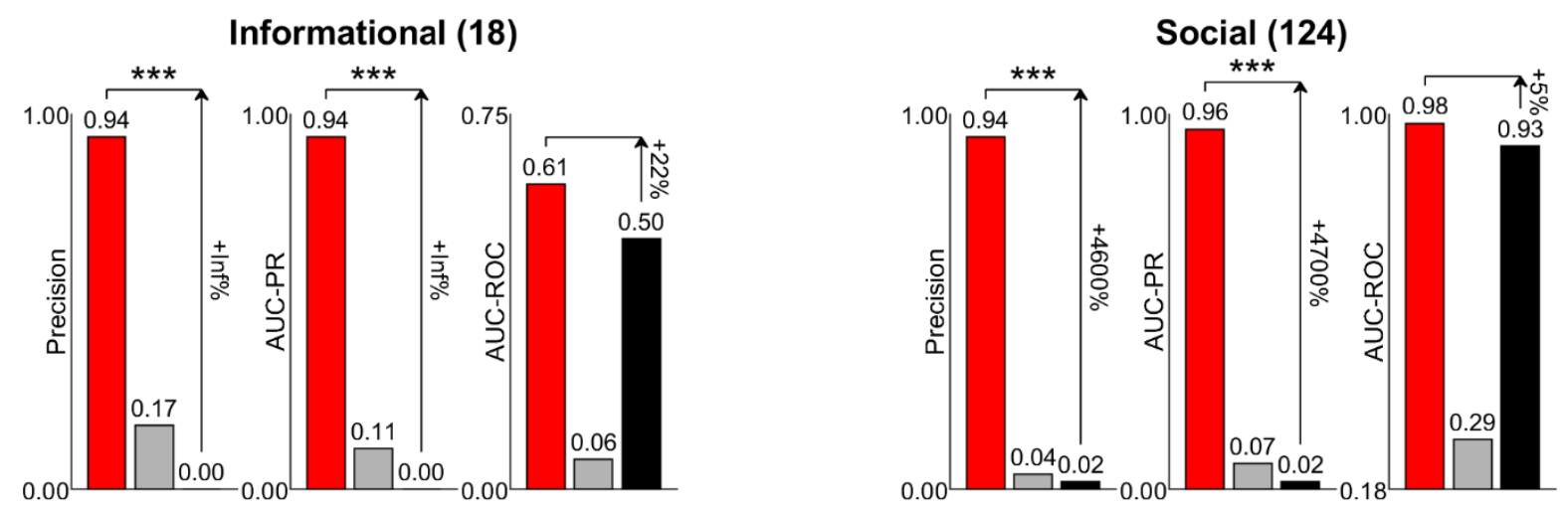

Transportation (35)
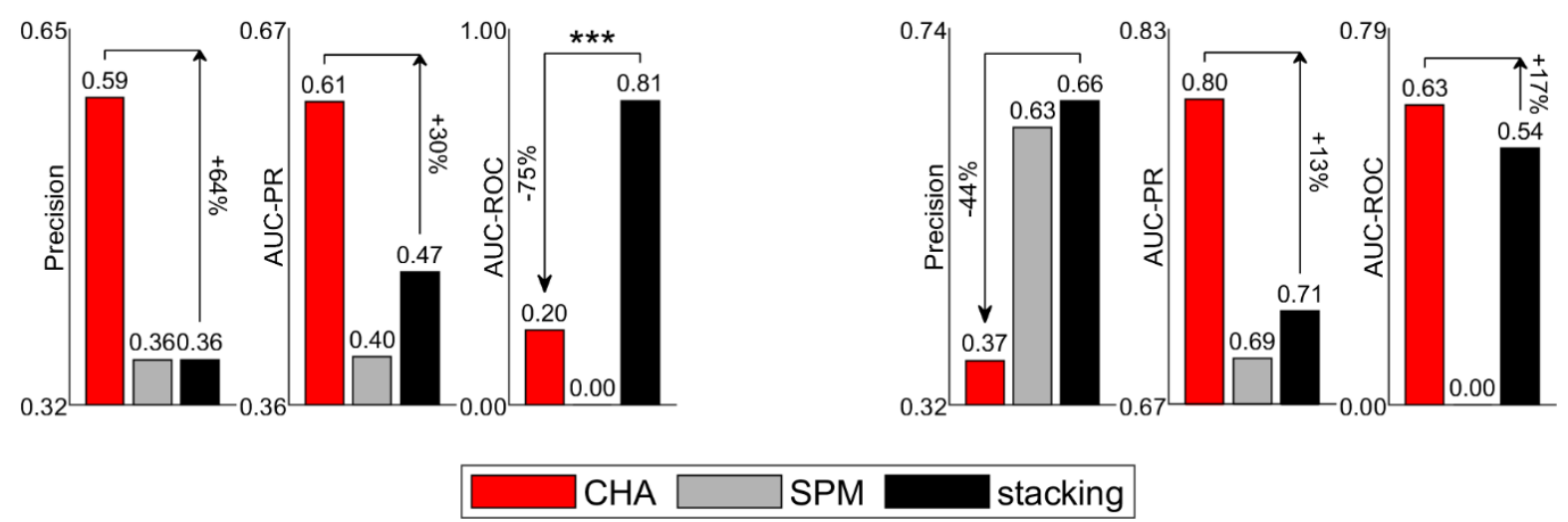

Figure 2. The barplots report, for each network domain (biological, economic, informational, social, technological, transportation) and for each evaluation measure, the win rate over the networks (whose number is indicated in brackets) of that domain for CHA, SPM and stacking. The arrows highlight the percentage of improvement of CHA with respect to stacking as $\frac{\text { CHA-stacking }}{\text { stacking }} \cdot 100$. The asterisks represent the p-value significance of the binomial test, comparing the number of wins of $\mathrm{CHA}$ and stacking on the networks of that domain. Networks and code for stacking has been released by Ghasemian et al. 1 at: https://github.com/Aghasemian/OptimalLinkPrediction. 